\title{
Matter as a Flow of Random Elementary Events in the Context of Discrete Time
}

\author{
Alexander Zaslavsky \\ Department of Information Technology, National Mining University, Dnepr, Ukraine \\ Email:am-47@mail.ru, amz.nmu@gmail.com
}

How to cite this paper: Zaslavsky, A. (2017) Matter as a Flow of Random Elementary Events in the Context of Discrete Time. Open Access Library Journal, 4: e3444.

https://doi.org/10.4236/oalib.1103444

Received: February 7, 2017

Accepted: March 3, 2017

Published: March 6, 2017

Copyright $\odot 2017$ by author and Open Access Library Inc.

This work is licensed under the Creative Commons Attribution International License (CC BY 4.0).

http://creativecommons.org/licenses/by/4.0/ (c) (i) Open Access

\begin{abstract}
The paper concerns certain consequences of discrete time hypothesis including physical regularities of a particle representation in the form of a flow of elementary events. In the context of the proposed formal description, particle observability has been determined; dependence of observability on a particle energy value has been shown. In terms of the proposed approach, a statistical rationale was given to the relative amount of dark (unobservable) matter.
\end{abstract}

\section{Subject Areas}

Modern Physics

\section{Keywords}

Discrete Time, Heisenberg Algebra, Flow, Energy, Substitution of Frequency, Observability, Dark Matter

\section{Introduction}

What is time: Discrete set or continuum? The problem remains to be open. Classical mechanics, relativity theory and quantum physics rely upon ideas of continual time. However, ideas of discrete time are still popular subject of discussion [1] [2] [3] [4] [5]. The main efforts of the authors of these papers are focused on justifying the hypothesis of discreteness. Present paper concerns certain interesting consequences of this hypothesis. If a particle approves itself within the outside world non-continuously it means that the particle can be represented by means of a sequence of elementary events which range of definition is countable set of time moments. As a rule, such a sequence is identified as a flow of events. In the general case, a flow of elementary events is a sequence of all possible random points within time axis with random gaps dividing them. In the context of these random points, by some means or other a particle approves 
itself in the universe. Flow formalism proposed in the paper opens the door to analyze temporal characteristics of matter as a random process which states follow each other in the form of the simplest (Poissonian) flow of events. States of a quantum particle are described with the help of a wave function within continual time. States of a particle-flow have been determined in terms of discrete time. In the both cases being just different representation forms, states of one and the same particle are considered. Hence, a mechanism to transform certain states into other ones should be available. Specifically states preset during discrete time moments should help reactivate wave function uniquely; moreover, discrete states themselves should be samples of the preset wave function. Similar mechanism is known in a theory of time series as sampling (Nyquist) theorem [6] [7] and [8]. Its application to the analysis of flows of events of high-energy particles forecasts a phenomenon which was not studied previously that is substitution of frequency (energy, mass) of a particle [9]. The phenomenon lies in the fact that after the energy (frequency) of particle exceeds a certain level (i.e., the Nyquist limit), the particle stops to be unambiguous interpretation. With different probability degrees, measuring process may determine two different values of frequency (energy, mass). It looks like a pair of random particles (initial particle and that dual to it) originates replacing it.

\section{Particle-Flow in the Context of Discrete Time}

Using formal description of deformed Heisenberg algebra [5], has obtained time component of deformed Schrödinger equation with accuracy up to the first-order of deformation parameter $\alpha$ in the form of

$$
j \hbar \partial_{t} \psi+\hbar^{2} \alpha \partial_{t}^{2} \psi=E \psi,
$$

where $E$-energy of the particle. Solution of the equation for wave function $\psi$ specified within arbitrary time interval $T$ with boundary conditions $\psi(0)=\psi(T)=0$ obtained by the authors of the mentioned paper means that time is quantized

$$
T=\tau \pi \frac{2 \alpha \hbar}{\sqrt{1-4 E \alpha}}, \quad \tau=0,1,2, \cdots
$$

We are interested in the consequences arising from the conclusion. In the context of inertial frame of reference connected with a particle under study (that is such frame of reference in terms of which the particle is fixed) its proper energy $E$ is equal to rest energy $E_{0}$. Classical Schrödinger equation applied by authors of paper [5] describes wave function the more accurate the less is the particle velocity to compare with light velocity. Thus, expression (2) delivers exact value of quantized time when rest energy of the particle $E_{0}$ is inserted. Substituting $\tau=1$ and $E=E_{0}$ into Equation (2) determine $\Delta t_{0}^{\prime}$ a quantum of proper time of a particle

$$
\Delta t_{0}^{\prime}=\pi \frac{2 \alpha \hbar}{\sqrt{1-4 E_{0} \alpha}}=\frac{a}{\sqrt{1-4 a \frac{E_{0}}{E_{P l}}}} t_{P l}
$$


where $a=\alpha E_{P l}$ is dimensionless constant specifying ratio between a quantum of proper time of a particle with zero rest mass and Planck time $t_{P l}$, and $E_{P l}$ is Planck energy. In [9] there is an attempt to substantiate the constant value being equal $a=3.648676282 \times 10^{-3}$. The value is a half of fine-structure constant. It is determined by the maximum possible protons energy $\sim 10^{20} \mathrm{eV}$.

A flow of elementary events in terms of intrinsic frame of reference connected with specific particle is regular by definition. It means that time intervals between events are exact and equal to the determined non-random $\Delta t_{0}$ value. This implies the following: clocks within reference system connected with a particle selected as reference body are synchronized with a flow of elementary events of the particle.

Consider the simplest flows of elementary events. The flows are ordinary ones having no aftereffect. The former means that probability for two or more elementary events of the particle under study to get into $\Delta t \rightarrow 0$ area is negligible to compare with probability for one event to get into it (the events are not simultaneous). The latter means that future time moments of the occurrence of events of a flow with the help of which a particle is represented cannot depend on the moments the event took place in the past.

Mathematical expectation $M[\tau(t, \Delta t)]$ of random $\tau(t, \Delta t)$ number of elementary events within $\Delta t$ interval being determined by the expression

$$
M[\tau(t, \Delta t)]=\int_{t}^{t+\Delta t} \lambda(t) \mathrm{d} t
$$

Value $\lambda(t)$ is the intensity (density) of the flow of elementary events of the particle being observed. This value can also be considered as the frequency of time discretization of the particle being observed. As shown in [9]

$$
\lambda(t)=\frac{\beta(t)}{\Delta t_{0}^{\prime}},
$$

where $\Delta t_{0}^{\prime}$-quantum proper time observed particle, $\beta(t)=E_{0} / E$.

Using Equation (3) determine flow density of elementary events of the particle being observed.

$$
\lambda(t)=\frac{\beta(t)}{a t_{P l}} \sqrt{1-4 a \frac{E_{0}}{E_{P l}}} .
$$

Within the simplest flow with $\lambda(t)$ density number of events $X(t, \theta)$ fallen on the time period with $\theta$ duration has Poisson distribution $P\{X(t, \theta)=k\}$ with the parameter

$$
\begin{aligned}
& \eta(t, \theta)=\int_{t}^{t+\theta} \lambda(t) \mathrm{d} t \\
& P\{X(t, \theta)=k\}=\frac{\eta^{k} e^{-\eta}}{k !}, \quad(k=0,1,2, \cdots)
\end{aligned}
$$

Function

$$
\bar{F}(\theta, m)=\exp \left\{-\int_{t}^{t+\theta} \lambda(t) \mathrm{d} t\right\}=\exp \left\{-\int_{t}^{t+\theta} \frac{\beta(t)}{a t_{P l}} \sqrt{1-4 a \frac{E_{0}}{E_{P l}}} \mathrm{~d} t\right\}
$$


is the probability of the fact that no state of a particle with $m=\frac{E_{0}}{c^{2}}$ mass within

$\theta$ time period can be detected $(k=0)$.

Consequently, probability $F(\theta, m)$ to find a particle with $m$ mass within $\theta$ time period is equal to

$$
F(\theta, m)=1-\exp \left\{-\int_{t}^{t+\theta} \frac{\beta(t)}{a t_{P l}} \sqrt{1-4 a \frac{E_{0}}{E_{P l}}} \mathrm{~d} t\right\} .
$$

In case of free particle $\beta(t)=$ const

$$
F(\theta, m)=1-\exp \left\{-\frac{\beta \theta}{a t_{P l}} \sqrt{1-4 a \frac{E_{0}}{E_{P l}}} \mathrm{~d} t\right\} .
$$

\section{Observability of a Particle-Flow}

Quantum mechanics describes state of a particle by wave function. Its parameters (frequency and wave vector) characterizes particle as a material object possessing energy and impulse. The domain of wave function is expressed by continuous time. However, if particle is considered as a flow of elementary events, then the domain of wave function is expressed by discrete time and the very wave function being expressed by the multitude of sampled values belongs to the class of generalized functions. In this relation, the problem of how well sampled values represent wave function being determined by energy and particle momentum is of high topicality. To find the solution we should use sampling theorem. According to the theorem, in application to the process being described by $\psi(r, t)$ wave function determined within $-\infty<t<\infty$ infinite time period having $v=\frac{E_{0}}{h}$ fixed frequency, wave function can be recaptured according to the sampling only under condition that $\lambda^{-1}$ time period between sampling meets the inequality

$$
\lambda^{-1} \leq \frac{h}{2 E_{0}}
$$

If within $\theta=\frac{h}{2 E_{0}}$ observation interval the particle with $E_{0}$ energy-mass proves itself by more than one elementary event interval of its wave function quantization meets sampling theorem and the particle is recognized unambiguously.

If within the interval the particle with $E_{0}$ energy-mass does not prove itself by any elementary event, it means that it is not observable as the flow of elementary events distributed through time in more than $\theta$ interval cannot show ambiguously the fact that it is the particle with $E_{0}$ energy-mass being observed at the moment (wave function of the particle cannot be recaptured on the sampling basis uniquely). Taking into account the aforementioned thesis, we will call observability of the particle which energy-mass is equal to $E_{0}$ the probability of the fact that the particle will prove itself by more than one elementary event 
within $\theta=\frac{h}{2 E_{0}}$ observation period. While putting this $\theta$ value into (10) we obtain formula to calculate particle observability

$$
F(\mu)=1-e^{-\beta \frac{\sqrt{1-4 \mu}}{2 \mu}}
$$

where $\mu=a \frac{E_{0}}{E_{P l}}=a t_{P l} \frac{E_{0}}{h}$ is energy-mass of a fixed particle in terms of relative units relative to $\frac{E_{P l}}{a}$ basis. The dependence of particle observability upon its relative mass in terms of various values of relativistic multiplier is demonstrated on the Figure 1.

The observability tends to a zero when velocity tends to light velocity, and particle mass is more than a zero; the observability tends to a unit when velocity tends to light velocity and particle mass tends to a zero.

\section{Substitution of the Frequency of Particle Wave Function}

According to sampling theorem, $\psi(r, t)$ function can be recovered according to the sequence of functions $\psi(r, \tau)$ only if $\lambda$ is such frequency of time discretization when

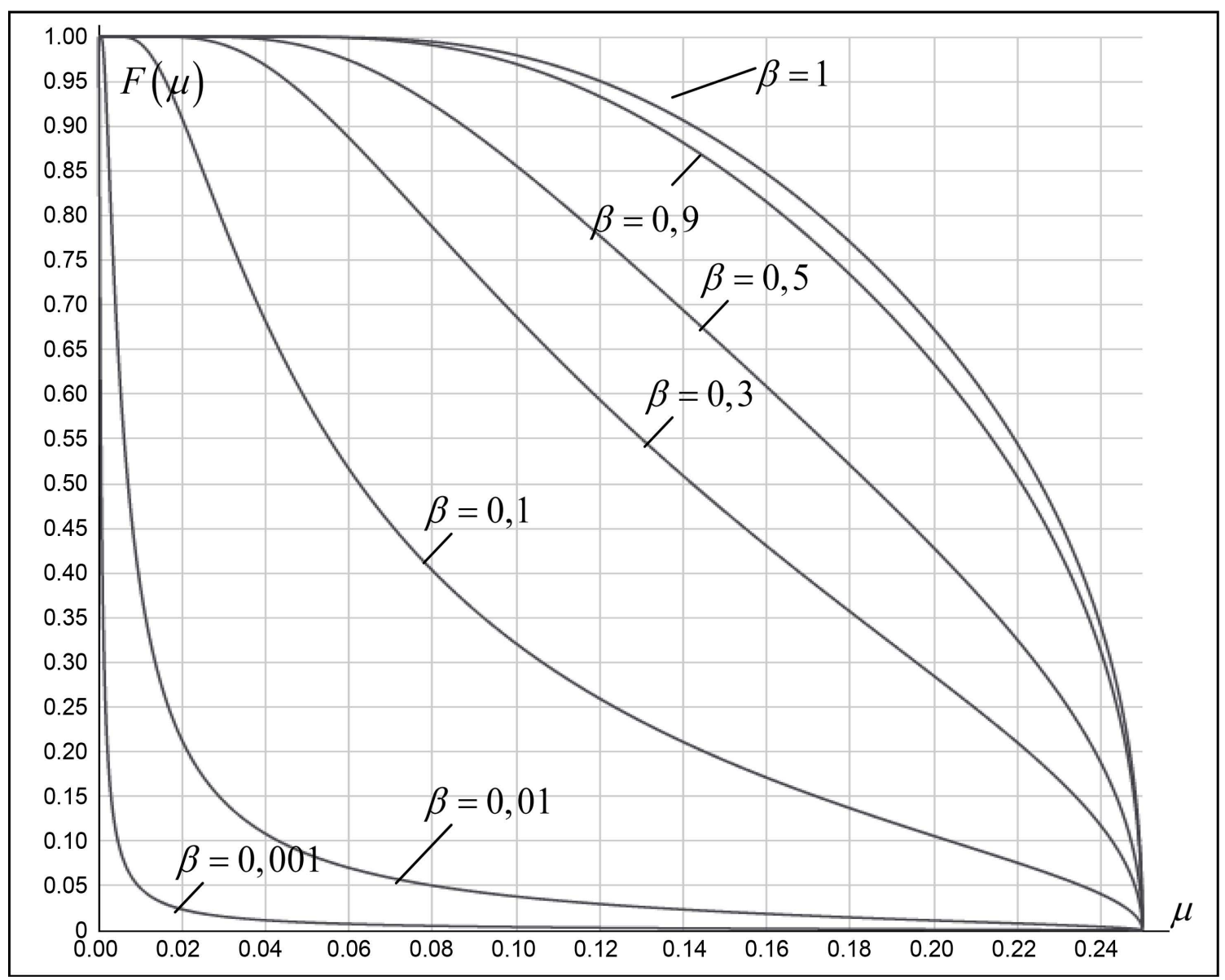

Figure 1. Dependence of particle observability upon its energy. 


$$
\frac{\omega}{2 \pi} \leq \frac{\lambda}{2}=F=\frac{\omega_{F}}{2 \pi}
$$

where $F$ and $\omega_{F}$ is Nyquist frequency.

Substituting $\frac{\omega}{2 \pi}$ frequency its corresponding value of particle energy $\frac{E}{h}$ as well as substituting value $\lambda$ from (6) we obtain inequality (13) in the form of

$$
\frac{\beta(t)}{a t_{P l}} \sqrt{1-4 a \frac{E_{0}}{E_{P l}}} \geq \frac{2 E}{h} \text {. }
$$

Substituting $\beta=\frac{E_{0}}{E}$ value into (14) we obtain the condition of single-valued recovering of a wave function according to its discrete samples

$$
\frac{E_{0}}{a t_{P l}} \sqrt{1-4 a \frac{E_{0}}{E_{P l}}} \geq \frac{2 E^{2}}{h} \text {. }
$$

The obtained result means following. Until particle energy exceeds value

$$
E_{F}=\sqrt{\frac{h E_{0}}{2 a t_{P l}} \sqrt{1-4 a \frac{E_{0}}{E_{P l}}}}
$$

its wave function being characterized by $\omega=\frac{E}{\hbar}$ frequency can be recovered unambiguously according to discrete series of instantaneous values following each other at $\lambda$ frequency.

If particle energy exceeds $E_{F}=\hbar \omega_{F}$ threshold, then as [9] shows there is a substitution of wave function frequency. Instead of a particle with

$E=\hbar \omega>\hbar \omega_{F}$ energy, one can find its dual particle with $E^{\prime}=\hbar \omega^{\prime}=2 E_{F}-E$ energy.

Taking into account the phenomenon of particle frequency substitution we have following ratio of wave function $\Omega$ frequency (which can take values either of $\omega$ initial frequency or $\omega^{\prime}$ dual one) and energy of the initial particle. The ratios can be considered as the extension of de Broglie equations to high-energy domains.

$$
\Omega= \begin{cases}\frac{E}{\hbar}, & E \leq \hbar \omega_{F}, \\ {\left[\left(\frac{E}{\hbar}-z \omega_{F}\right), \frac{E}{\hbar}\right],} & z=0, \\ {\left[\left((z+1) \omega_{F}-\frac{E}{\hbar}\right), \frac{E}{\hbar}\right],} & \\ {[(z=2,4, \cdots, \text { (even number) }} \\ \end{cases}
$$

where $Z$ is a multiplicity of Nyquist frequency exceedance.

Figure 2 demonstrates the diagram of wave function frequency substitution visualizing the ratio between the energy of initial and dual particles.

\section{Uncertainty of Particle Energy after Frequency Substitution}

Substitution of wave function energy means that when particle energy exceeds 


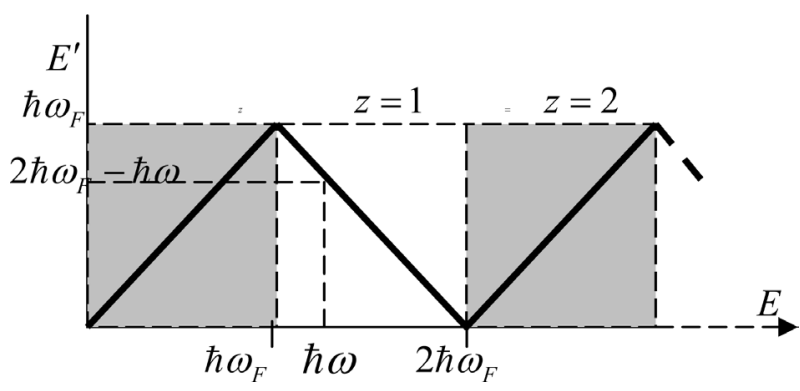

Figure 2. Diagram of wave function frequency substitution.

$E_{F}=\hbar \omega_{F}$ level its energy becomes many-valued. The many-validness should be manifested in further measurements, which can detect within time-space range where before there was only initial particle or only initial particle with $E$ energy, or only its dual particle with $E^{\prime}=2 \hbar \omega_{F}-E \leq \hbar \omega_{F}$ energy, or both particles.

Mark as $s_{0}$ the probability of the fact that particle energy after frequency substitution is equal to the energy of the initial particle before substitution. Mark probability of the fact that particle energy after frequency substitution is equal to the energy of dual particle of $z$ multiplicity as $s_{z}$. Two possible effects of frequency substitution are compatible (one does not excludes another) but independent (one does not result from another). Consequently, in this case condition of probabilities normalization is as follows

$$
s_{0}+s_{z}+s_{0} s_{z}=1 .
$$

Energy conservation law should not be violated in terms of frequency substitution. Assume that, for instance, there are $N$ of initial particles each having $\hbar \omega$ energy. If the energy exceeds $\hbar \omega_{F}$ threshold, there is a substitutions of frequency of all the particles. Owing to energy many-valuedness beyond the threshold of frequency substitution we will find $N_{0}=\left(s_{0}+s_{0} s_{z}\right) N$ of particles with $E$ initial energy and $N_{z}=\left(s_{z}+s_{0} s_{z}\right) N$ with $E^{\prime}$ dual energy. Energy conservation law requires the total energy of the particles to be equal to the total energy of the initial particles. Consequently, energy of the initial particle should be equal to the sum of mathematical expectation of initial particle energy after frequency substitution and mathematical expectation of dual particle energy.

\subsection{Energy Law Conservation in Terms of Odd Multiplicity Frequency Substitution}

$$
\hbar \omega=\left(s_{0}+s_{0} s_{z}\right) \hbar \omega+\left(s_{z}+s_{0} s_{z}\right)\left[(z+1) \hbar \omega_{F}-\hbar \omega\right] .
$$

While solving (18) and (19) together, we will find $s_{0}$ and $s_{z}$ probabilities in terms of odd frequency substitution

$$
\begin{gathered}
s_{0}=\left[\left(\frac{\omega}{(z+1) \omega_{F}-\omega}-1\right), 1\right], \\
s_{z}=\frac{1-s_{0}}{1+s_{0}}=\left[\left(\frac{2(z+1) \omega_{F}}{\omega}-3\right), 0\right] .
\end{gathered}
$$


From $0 \leq s_{0}<1$ condition we obtain range of energy values

$$
\frac{(z+1)}{2} \omega_{F} \leq \omega<\frac{2(z+1)}{3} \omega_{F} .
$$

As it is seen from the inequality, $0 \leq s_{0}<1$ condition is met for odd $z$ values if only $z=1$.

If $z=3,5, \cdots$, then frequency takes $\omega \geq 3 \omega_{F}, \omega \geq 5 \omega_{F}, \cdots$ values; to meet $0 \leq s_{0}<1$ condition, $\omega<2 \frac{2}{3} \omega_{F}, \omega<4 \omega_{F}, \cdots$ is required respectively.

\subsection{Energy Law Conservation in Terms of Even Multiplicity Frequency Substitution}

$$
\hbar \omega=\left(s_{0}+s_{0} s_{z}\right) \hbar \omega+\left(s_{z}+s_{0} s_{z}\right)\left[\hbar \omega-z \hbar \omega_{F}\right] .
$$

While solving (18) and (23) together, we will find $s_{0}$ and $s_{z}$ probabilities in terms of substitution of even multiplicity frequency.

$$
\begin{gathered}
s_{0}=\left[\left(\frac{z \omega_{F}}{\omega-z \omega_{F}}\right), 1\right] . \\
S_{z}=\left[\left(1-2 \frac{z \omega_{F}}{\omega}\right), 0\right] .
\end{gathered}
$$

From $0 \leq s_{0}<1$ condition we will obtain range of energy values

$$
\omega>2 z \omega_{F} \text {. }
$$

As $z$ is an even integer redoubling after frequency exceeds $\omega \geq(z+1) \omega_{F}$ value, then it is impossible for (26) to be valid for any $z>0$ values. Thus, if $z=0$, then there is no frequency substitution by convention and the obtained result means that the substitution at the even multiplicity frequency cannot be detected. Dual particles can be generated only within frequency range from $\omega_{F}$ to $4 / 3 \omega_{F}$. Figure 3 shows dependences of $s_{0}$ and $s_{z}$ probabilities upon wave function frequency.

\section{Evaluation of the Number of Nonobservable (Dark) Matter}

As we can see from the abovementioned, Matter is not quite observable. Particle observability $F(\mu)$ is a conditional (a posteriori) probability of detecting the

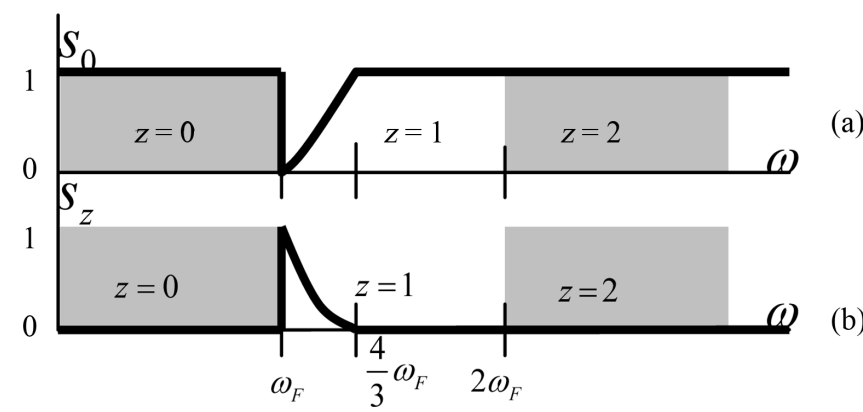

Figure 3. Range of probabilities of wave function frequency substitution: (a) initial particle probability, (b) dual particle probability. 
particle if its mass is equal to $\mu$. Assume that mass of the initial particle with $s_{0}+s_{0} s_{z}$ a priori probability is equal to $\mu$. Total probability $P(\mu)$ of the fact that the initial particle will be detected is equal to the product of a prior probability by a posteriori probability

$$
P(\mu)=\left(s_{0}+s_{0} s_{z}\right) \cdot F(\mu) .
$$

Consider linearly ordered series $R(\mu)$ of possible relative masses of $(\beta \approx 1)$ nonrelativistic particles

$$
R(\mu)=\left(\mu_{0}, \mu_{1}, \cdots, \mu_{F}, \mu_{F 1}, \cdots, \mu_{\text {sup }}\right),
$$

Were $\mu_{0}<\mu_{1}<\cdots<\mu_{F}<\mu_{F 1}<\cdots<\mu_{\text {sup }}$. The series is limited by $\mu_{\text {sup }}=0,25$ maximum possible mass ratio that arises from formula (3). The series has value of $\mu_{F}$ mass beginning from which substitution of frequency - mass - energy, appears. Find the mass from (15) substituting value $\mu_{F}$ instead of $a \frac{E_{0}}{E_{P l}}$ and $a t_{P l} \frac{E}{\hbar}$

$$
\sqrt{1-4 \mu_{F}}=2 \mu_{F} .
$$

While solving the equation we obtain $\mu_{F}=\frac{1}{2}(\sqrt{2}-1) \approx 0,207$.

Mass substitution means that the particle with $\mu>\mu_{F}$ mass can prove itself as the particle with $2 \mu_{F}-\mu<\mu_{F}$ mass.

The domain of mass (frequency) values within which substitution can take place, as it is shown before (22) is between $\mu_{F}$ and $\frac{4}{3} \mu_{F}=0,273$ values. However, due to the fact that maximum possible particle mass is less than the upper limit determined from $s_{0}<1$ condition, the domain is limited at the top by $\mu_{\text {sup }}=0,25$ limit mass value.

Initial particles "leave" range of heavy particles due to frequency substitution and "move", becoming dual ones, to the range of medium-mass and light particles. In this context owing to energy conservation (19) total mass of all the particles in the universe does not change.

Use mean (averaged in terms of all the masses) value of total particle observability possibility as an approximate estimation of the ratio of the observable particles masses to the masses of all the particles

$$
\langle P(\mu)\rangle=\frac{1}{\mu_{\text {sup }}} \int_{0}^{\mu_{\text {sup }}}\left(s_{0}+s_{0} s_{z}\right) \cdot F(\mu) \mathrm{d} \mu .
$$

Then we obtain the estimation of $\mu_{D M}$ relative mass of material (large), cold $\beta \approx 1$ nonobservable particles in the form of

$$
\mu_{D M}=\frac{1}{\mu_{\text {sup }}} \int_{0}^{\mu_{\text {sup }}}\left[1-s_{0}\left(1+s_{z}\right) \cdot F(\mu)\right] \mathrm{d} \mu .
$$

Inserting previously determined values of limits of ranges of masses of initial particles as well as prior probabilities and posterior probabilities we obtain 


$$
\begin{aligned}
\mu_{D M}= & 4 \int_{0}^{0,207} e^{-\frac{\sqrt{1-4 \mu}}{2 \mu}} \mathrm{d} \mu+4 \int_{0,207}^{0,25} \mathrm{~d} \mu- \\
& -\int_{0,207}^{0,25}\left[8 \frac{\mu-0,207}{0,414-\mu}\left(\frac{0,828}{\mu}-2\right)\left(1-e^{-\frac{\sqrt{1-4 \mu}}{2 \mu}}\right)\right] \mathrm{d} \mu \approx 0,22 .
\end{aligned}
$$

Thus, theoretical estimation of relative amount of nonobservable (dark) cold matter with reference to the whole energy being mass of universe is almost $22 \%$ which is equivalent of the available empirical data (20\% ... 30\%).

\section{Conclusions}

Matter being considered as a flow of elementary events which frequency of occurrence is restricted due to time discreteness can be observed in part only.

It should be noted that the square of wave function amplitude does not fit the observability properly. It is the density of probability to find a particle at $t$ moment within the given spatial point under condition that the particle is observable, i.e. it is well known that it occurs within some area of universe. This condition is represented mathematically by normalization of wave function. Observability is the probability that the particle of the specified energy is present (somewhere) in the universe. As for the observability, it is the probability that a particle of preset energy is available somewhere in the universe. Observability value of the particle decreases along with the increase of its energy.

Dark matter is that its part which cannot be observable principally owing to time discreteness.

Time quantization applies restrictions upon the value of maximum allowable particle mass at which time interval between elementary events becomes indefinitely great. As a result, particles with the maximum large mass are not observable both at rest and at any movement velocity.

\section{Acknowledgements}

The author thanks Larissa Tokar for assistance in preparing the paper, Stanislav Lisnyak for meaningful discussion, and reviewer for helpful comments.

\section{References}

[1] Esposito, S. and Salesi G. (2009) Fundamental Times, Lengths and Physical Constants: Some Unknown Contributions by Ettore Majorana. arXiv: 1001.0050v1 [physics. hist-ph].

[2] Amelino-Camelia, G. (2000) Relativity in Space-Times with Short-Distance Structure Governed by an Observer-Independent (Planckian) Length Scale. arXiv:gr-qc/0012051v2.

[3] He, G.P. (2009) An Experimentally Testable Proof of the Discreteness of Time. arXiv: 0911.2416v2 [quant-ph].

[4] Faizal, M., Ali, A.F. and Das, S. (2014) Discreteness of Time in the Evolution of the Universe. arXiv: $1411.5675 \mathrm{v} 1$ [gr-qc].

[5] Faizal, M., Khalil, M.M. and Das, S. (2016) Time Crystals from Minimum Time uncertainty. The European Physical Journal C, 76, 30. 
https://doi.org/10.1140/epjc/s10052-016-3884-4

[6] Nyquist, H. (1928) Certain Topics in Telegraph Transmission Theory. Transactions of the American Institute of Electrical Engineers, 47, 617-644.

https://doi.org/10.1109/T-AIEE.1928.5055024

[7] Kotelnikov, V.A. (2006) Bandwidth Ether and Wire in Electrical CommunicationUnion Energy Committee. Materials to the I All-Union Congress on the Technical Reconstruction of Communication and Development of Low-Current Industry 1933. Reprint: Physics-Uspekhi, 176:7, 762-770.

[8] Shannon, C. (1949) Communication in the Presence of Noise. Proceedings of the IRE, 37, 10-21.

[9] Zaslavsky, A. (2015) Anomalous Behavior of Ultra-Relativistic Particles-A Phenomenon That Can Confirm the Discreteness of Time. American Journal of Modern Physics, 4, 34-40.

Submit or recommend next manuscript to OALib Journal and we will provide best service for you:

- Publication frequency: Monthly

- 9 subject areas of science, technology and medicine

- Fair and rigorous peer-review system

- Fast publication process

- Article promotion in various social networking sites (LinkedIn, Facebook, Twitter, etc.)

- Maximum dissemination of your research work

Submit Your Paper Online: Click Here to Submit

Or Contact service@oalib.com 